\section{Local priorities can be too parochial for biodiversity}

I cannot accept the provocative point made by R. J. Smith and colleagues in their Opinion article (Nature 462, 280-281; 2009) that decision-making for conservation research and implementation priorities should be left to local people.

Speaking mainly from experience in North America, I believe that most local agencies (for example, US county governments) have little concept of what biodiversity is, much less how to measure, prioritize or protect it. Local land-use planning tends to be parochial and is divorced from conservation planning, which occurs on a broader geographical scale (see Lasting Landscapes, Environmental Law Institute, 2007).

Local governments often allow development in biodiversity-rich and ecologically sensitive areas, even when it is demonstrably unnecessary. In Florida, huge subdivisions and condominium complexes can sit vacant for months or even years.

This irrational development is encouraged because local agencies are controlled by politicians and powerful economic interests. They care about species conservation only when the species in question is protected under state or federal law or if it is hunted; unlisted and non-game species (especially unvertebrates) receive scant attention. The situation is aggravated because local agencies lack appropriately trained personnel for managing ecosystem conservation.

Local agencies' capabilities are likely to be even more of a problem in developing countries. Letting them set the conservation agenda by themselves could therefore be a mistake.

Academic researchers, conservation non-governmental organizations and other 'foreign' interests tend to be better informed, less subject to local

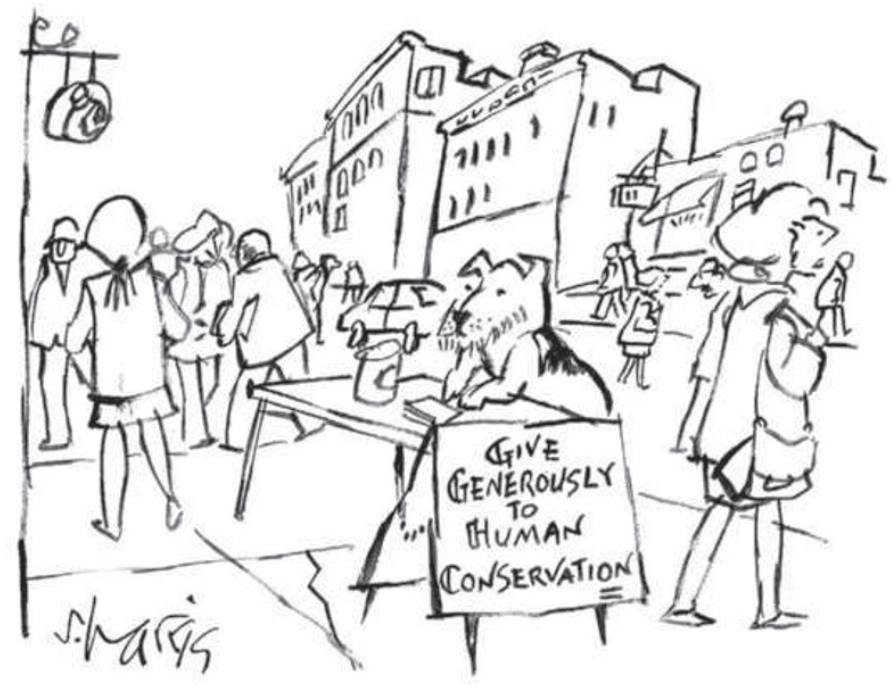

political influence and more experienced in conservation planning than local agencies. Let's allow these parties to work together towards mutual conservation goals, rather than radically switching from hegemony by the broad thinkers to hegemony by parochialists. Reed F. Noss Department of Biology, University of Central Florida, Orlando, Florida 32816-2368, USA

e-mail: rnoss@mail.ucf.edu

\section{Psychiatry: medicine benefits from cultural and personal insights}

Anthropology is a valuable component of the multidisciplinary research effort into psychiatric disorders that is recommended in your Editorial (Nature 463, 9; 2010).

Since the 1920s, anthropologists have helped psychiatrists consider the cultural factors influencing their patients, and psychiatrists have helped anthropologists focus on the individual in their societal studies. Anthropologists have published important ethnographic texts on such topics as the structure and culture of psychiatric hospitals, patients' transition to community life after being deinstitutionalized, and the shifting historical circumstances that contribute to both professional and personal understandings of psychiatric disorders.

Too often dismissed as merely 'anecdotal', anthropology emphasizes the lived experiences of individuals with psychiatric disorders - experiences that rarely figure in research protocols and quantitative data. Elyn Saks, an associate dean and law professor at the University of Southern California, Los Angeles, who has schizophrenia, says that denial of mental illness is not a psychiatric symptom but a defence mechanism against the realization that there is something wrong with the way one perceives the world (E. R. Saks Am. J. Psychiatry 166, 972-973; 2009). By listening to the personal stories of different patients, anthropologists stumble upon observations such as these all the time. Anthropology offers the opportunity to bring individual voices into conversation with the psychiatrists and scientists who are focused on the bigger picture.

Although anthropological research may be limited in scope - the subject population often numbers only in the tens - the perspective of a medical outsider with a different analytical lens can reveal new avenues of inquiry that clinical studies, in their need to conform to the standards of evidence-based medicine, may overlook. Given the limited knowledge of the biology underlying psychiatric disorders, it is time to open up the research agenda to disciplines that think outside the medical box.

Ellen Rubinstein Department of Anthropology, Yale University, New Haven, Connecticut 06511, USA e-mail: ellen.rubinstein@yale.edu

\section{Psychiatry: Brazil debates dismantling all mental hospitals}

Your Editorial on psychiatric disorders (Nature 463, 9; 2010) mentions the misplaced stigma often associated with them. In Brazil, there is strong opposition within certain social sectors to the idea of mental-health interventions. A proposed law to dismantle all psychiatric hospitals in the country has been arousing fierce debate since 2001.

One side argues that the law would end the widespread abandoning of the poor in psychiatric institutions, a practice that has been criticized as detrimental to the patients' chances of long-term recovery. Opponents, however, say that it would eliminate the opportunity for immediate care of acutely affected patients, such as those with suicidal depression or delusional schizophrenia; this would increase the risks for both patients and their families, while placing all the responsibility on the latter.

This debate reflects the fact that, in many countries, the necessary political and methodological changes must be conceived of as part of a multistage programme, with an emphasis on ethics and halting human-rights violations. If these measures had been taken in the past, this uncomfortable discussion would not be necessary and Brazil could focus on strengthening the science underlying its health-care system. Álvaro Machado Dias Department of Neuroscience and Behaviour, Institute of Psychology, University of São Paulo, São Paulo, Brazil e-mail: alvaromd@usp.br 